\title{
Demonstration of a Gimbal Mounted, High Resolution Charge Coupled Device (CCD) Television Camera in Lieu of Direct View Optics for Air to Ground Targeting
}

\author{
Major Paul C. Schreck, USMC \\ Naval Rotary Wing Aircraft Test Squadron \\ 22755 Saufley Rd. Unit 1 \\ Patuxent River, MD 20653-1619 \\ (301) $342-1768$ \\ schreckpc\%am4@mr.nawcad.navy.mil
}

\begin{abstract}
The process of air to ground targeting for military attack aircraft involves three distinct, sequential phases of visual discrimination: detection, recognition, and identification (DRI). The intent of this paper is to discuss the tactical merits of charged coupled device (CCD) technology in air to ground target DRI, while contrasting the limitations imposed by the loss of direct view optics (DVO). During an extensive flight and ground test program conducted at the Naval Air Warfare Center Aircraft Division, Patuxent River, MD from October, 1996 to February 1997, an experimental airborne electo-optical targeting sensor suite featuring high resolution $\mathrm{CCD}$ cameras was evaluated by direct comparison to an existing system based on DVO. The results indicated superior performance of the CCD cameras in low ambient lighting conditions, but the absence of color was a major hindrance to rapid target area acquisition. Test results indicated that high resolution and magnification are not the most critical design criteria for an airborne electo-optical targeting suite; human factors must influence the design based upon a thorough, systematic analysis conducted during flight test.
\end{abstract}

\section{TABLE OF CONTENTS}

1. INTRODUCTION

2. SCOPE AND METHOD OF TEST

3. DATA ANALYSIS

4. RESULTS AND EVALUATION

5. CONCLUSIONS

\section{INTRODUCTION}

Night Targeting System (NTS)

The Bell Helicopter Textron, Inc. AH-1W SuperCobra is a single main rotor, two place aircraft with tandem seating that serves as the primary attack helicopter platform for the United States Marine Corps (USMC). Capable of employing a wide range of unguided and precision guided munitions, the AH-IW is often touted as the world's most versatile attack helicopter (Fig. 1).

The sensor suite currently installed on the $\mathrm{AH}-1 \mathrm{~W}$ is the Night Targeting System (NTS) (Fig. 2). Designed by theTamam Division of Israel Aircraft Industries Electronics Group, and currently manufactured by Kollsman, Inc., the NTS is a modification to the M-65 Telescopic Sight Unit

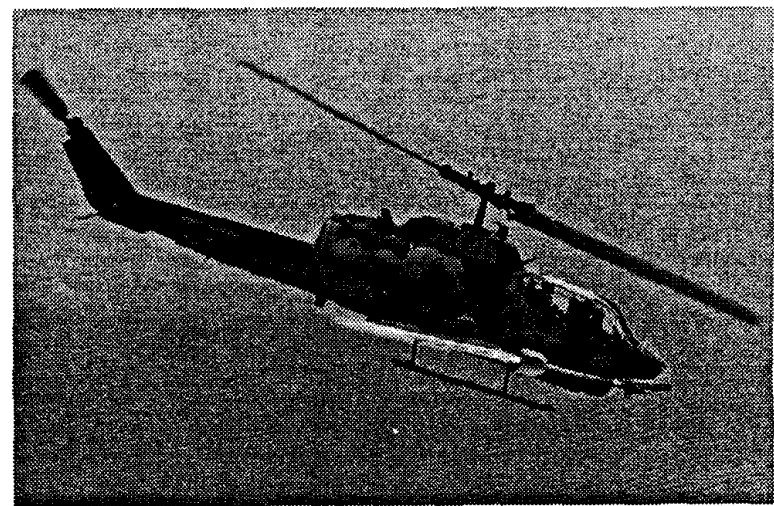

Figure 1. AH-1 W SuperCobra

fielded by Hughes Aircraft Corporation in the early 1970's. The M-65 employs a two field of view (FOV), direct view optics (DVO) system that can be slewed vertically and horizontally for target DRI. The two fields of view provide $2 \mathrm{X}$ (power) and $13 \mathrm{X}$ magnification to the operator in the front seat of the aircraft.

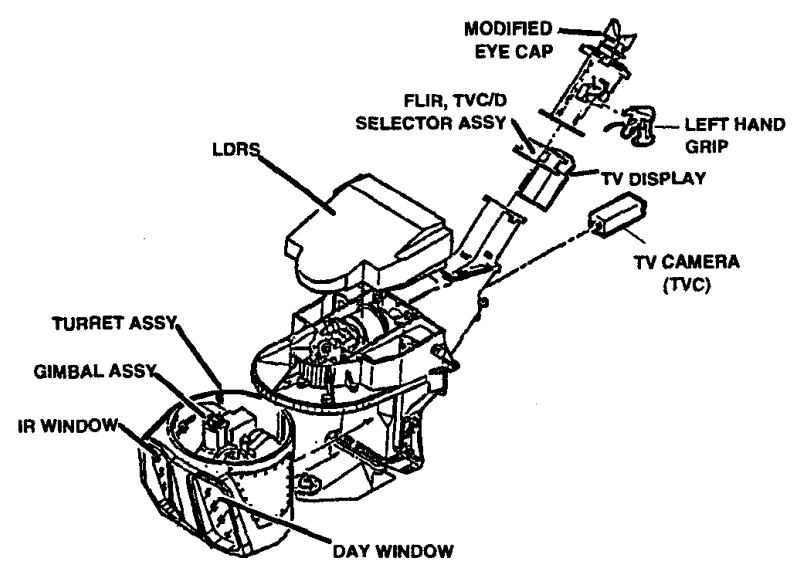

Figure 2. AH-1 W Night Targeting System

The NTS improved upon the basic M-65 by incorporating an onboard laser designator/rangefinder system (LDRS), a forward looking infrared (FLIR) sensor adjacent to the gimbal mounted DVO, and a CCD camera mounted within 
the M-65, approximately midway in the optical relay tube (ORT) that extends into the forward cockpit (Fig 3).

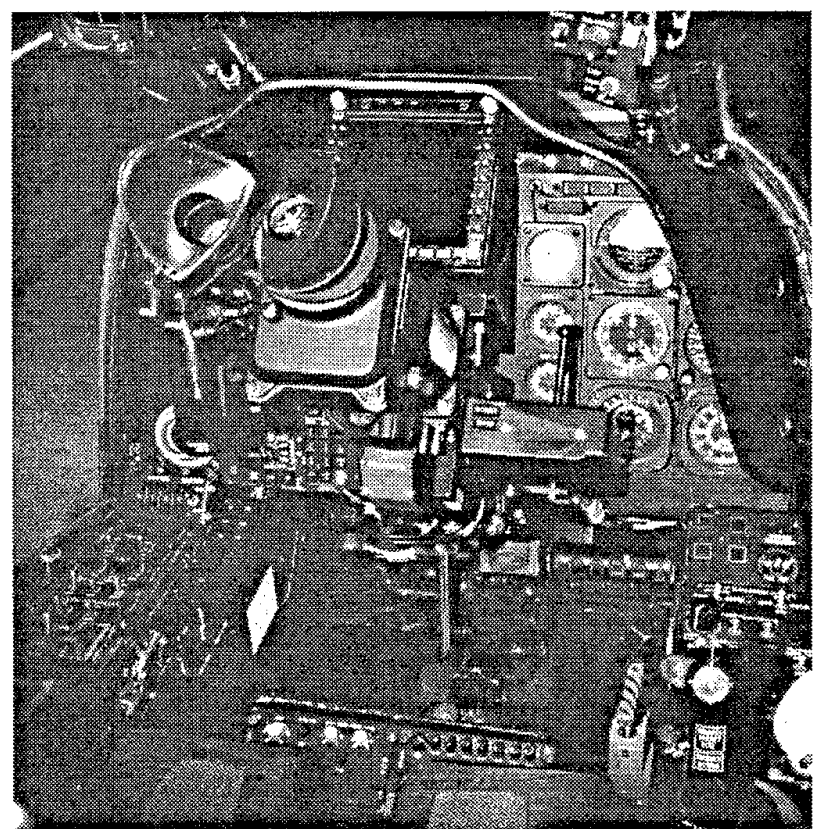

Figure 3. Forward Cockpit of NTS Equipped AH-1W

The optical train of the basic NTS provides a monocular output to the eyepiece lens. Two eyerests are provided on the user end of the ORT, one of which is blank and can be rotated so the operator can use either eye to look through the optics. An operator selectable, single wavelength laser filter is provided near the eyepiece end of the ORT. The CCD camera image is displayed to the operator on either a one square inch cathode ray tube (CRT) within the ORT, or on a five square inch CRT multifunction display (MFD). Both displays are monochromatic. The CCD camera's placement within the optical path provides that sensor with improved magnification over DVO. However, since the M-65/NTS optics attenuate at least $70 \%$ of the light available at the objective lens through the optical train, the low light level (i.e., dusk/dawn) performance of the DVO, along with the internal CCD camera, is degraded. Furthermore, the relatively low magnification of the DVO requires the attack helicopter pilot to close with the target to effectively complete the DRI process, reducing aircraft survivability by decreasing standoff from enemy weapons.

\section{Advanced Night Targeting System (NTS"A")}

The NTS"A" intended to improve upon the basic NTS by completely removing the DVO and replacing them with high resolution, gimbal mounted $C C D$ cameras. The NTS" A" incorporated two camera heads mounted on the M65 's slewable gimbal in the space vacated by the optics, adjacent to the FLIR sensor (Fig. 4).

Each CCD sensor was a monochromatic solid state imaging array of elements greater than $750(\mathrm{H}) \times 488(\mathrm{~W})$ in $1 / 2$ " format ( $8 \mathrm{~mm}$ diagonal). The ORT was removed from the forward cockpit, leaving only the existing five square inch monochrome MFD for sensor output to the operator. The MFD was repositioned for optimum viewing distance and a more normal line of sight to the design eye point than the NTS cockpit arrangement (Fig. 5).

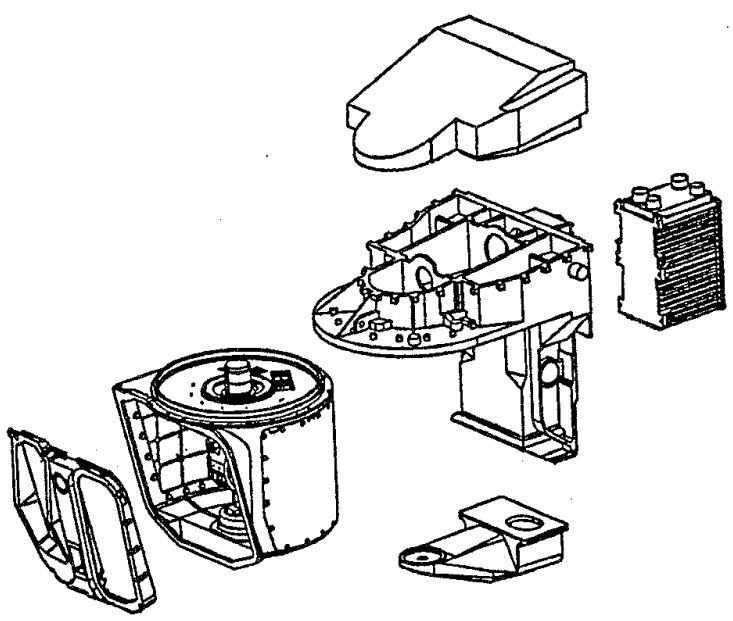

Figure 4. NTS"A" General Layout

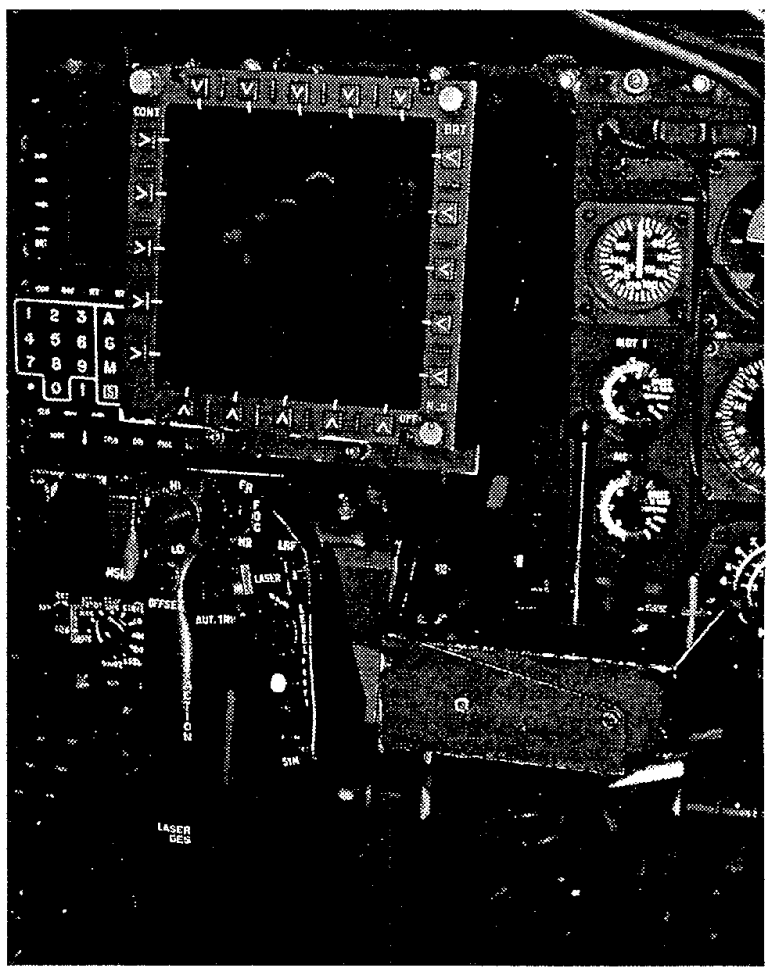

Figure 5. Forward Cockpit of NTS"A" Equipped AH-1W

The two camera head arrangement provided four fields of view and an order of magnitude increase in magnification. A basic comparison of sensor magnification is presented in Table 1.

The optical layout of the NTS"A" is presented in Figure 6. Light enters the slewable turret through the outer glass 
Table 1. System Design Magnifications

\begin{tabular}{|c|c|c|c|}
\hline \multirow{2}{*}{ Field of View } & NTS"A" & NTS DVO & NTS TVC \\
\cline { 2 - 4 } & \multicolumn{3}{|c|}{ (Field of View/Magnification) } \\
\hline $\begin{array}{c}\text { Electronic } \\
\text { Zoom Narrow }\end{array}$ & $0.45 \% / 132.8 \mathrm{X}$ & & \\
\hline $\begin{array}{c}\text { Narrow } \\
\text { (NFOV) }\end{array}$ & $0.9^{\circ} / 65.4 \mathrm{X}$ & $4.6 \% 12.8 \mathrm{X}$ & $2.0^{\circ} / 31.3 \mathrm{X}$ \\
\hline $\begin{array}{c}\text { Medium } \\
\text { (MFOV) }\end{array}$ & $3.5^{\circ} / 16.8 \mathrm{X}$ & & \\
\hline $\begin{array}{c}\text { Wide } \\
\text { (WFOV) }\end{array}$ & $18^{\circ} / 3.0 \mathrm{X}$ & $29.6^{\circ} / 2.3 \mathrm{X}$ & $12.5^{\circ} / 3.2 \mathrm{X}$ \\
\hline
\end{tabular}

window. The light is directed to the medium and wide field of view camera head through an objective lens. In MFOV, the light is then focused directly on the CCD. When selecting WFOV, additional optics are positioned between the objective and the CCD to expand the FOV. Both the WFOV and MFOV are focused at infinity (beyond $200 \mathrm{~m}$ ) and cannot be manually adjusted. Light arrives at the CCD in the NFOV camera head by traveling through an optical path consisting of several fold mirrors, lenses, and wedges. The narrow field of view can be manually focused by the user with the cockpit mounted controls. The narrow field of view can be zoomed by electronic doubling to twice its normal size with a corresponding loss in resolution.

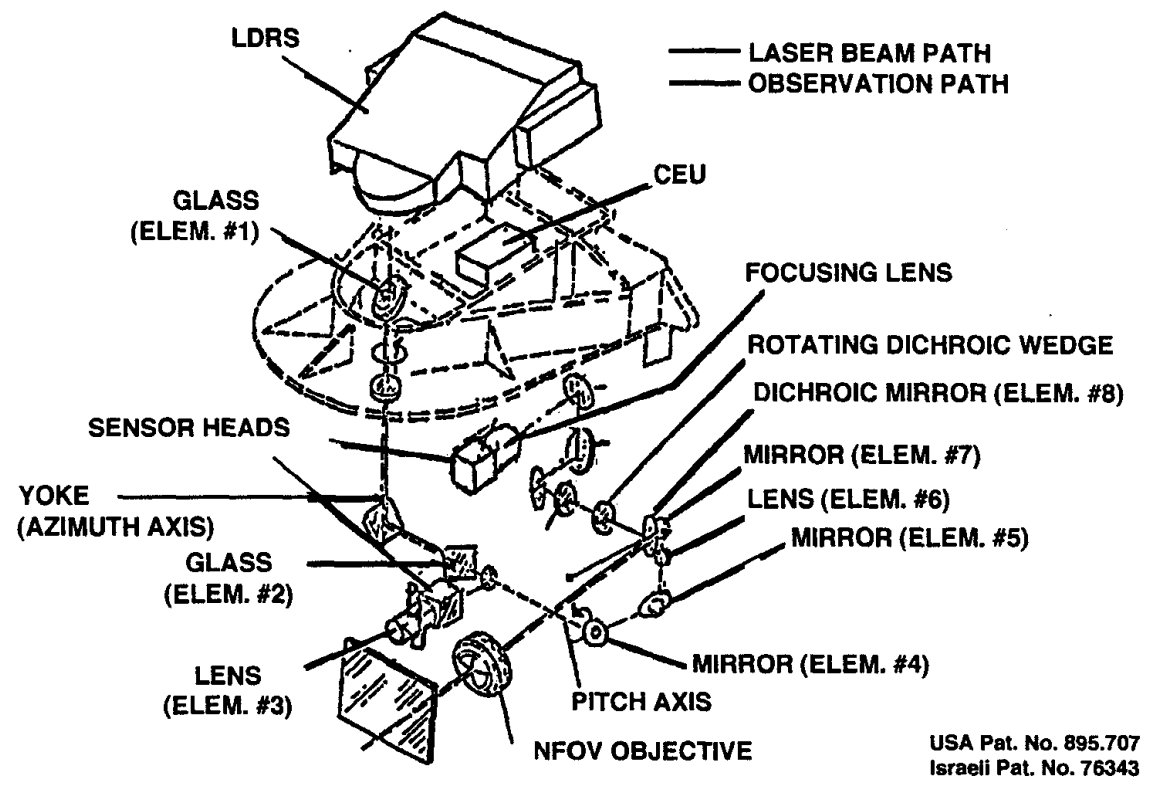

Figure 6. Optical Layout of the NTS"A"

The primary design detractors of the NTS drove the design of NTS"A". The NTS ORT in the forward cockpit is a major hindrance to rapid emergency egress, as well as a serious impact hazard to the forward cockpit occupant during forced or hard landings. An interim solution to this problem was the development of the inflatable body and head restraint system (IBAHRS). IBAHRS employs cartridge activated, deceleration triggered air bags in the shoulder harness of the five point restraint system in both cockpits. Another limitation of the NTS is the potential for laser inflicted eye damage when utilizing the DVO. The laser filter originally incorporated in the M-65 optics is single wavelength and does not provide adequate protection against all laser threats on the modern battlefield.

\section{NTS"A" Optical Design}

The NTS" $A$ " cameras were designed to exploit a wider range of the electromagnetic (EM) spectrum than the NTS DVO and camera. The day sky contains a wide distribution of the EM spectrum, from cosmic rays to power transmission emissions. The human eye can only detect the spectrum of visible light, from about 380 to 780 nanometers (nm). As the sun nears and eventually descends below the horizon causing a decrease in total illuminance, the luminance from the visible wavelengths decreases more rapidly as compared to the IR wavelengths. The eye then shifts to scotopic vision, utilizing the rods in the retina for target detection and becoming most sensitive to wavelengths around $500 \mathrm{~nm}$. Since the optical path of the NTS attenuates approximately $70 \%$ of the light striking the objective lens, the optics are essentially worse than the unaided eye for target detection in low light level, i.e., dusk and dawn, conditions. This same design limitation prevents the NTS camera from capitalizing on the increased sensitivity of the CCD, from about 200 to $1100 \mathrm{~nm}$, which includes the near IR region. The NTS"A" cameras were designed to minimize optical losses and take full advantage of the near IR sensitivity of the CCD's by placing them on gimbal, where maximum light can be directly focused with a potentially drastic increase in sensor performance. The responsivity of the CCD to near infrared wavelengths was designed to improve sensor performance through obscurants (e.g., dust, haze, smoke, etc.) that reduce atmospheric transmission. Since the particulates suspended in those obscurants are generally large enough to reduce the 
luminance of the visible spectrum through scattering, absorption, or refraction, they are less effective versus near IR wavelengths. Both systems incorporate a $1064 \mathrm{~nm}$ Neodymium YAG laser used for designating targets for laser guided munitions and rangefinding. Both NFOV optical trains contain provisions for directing the laser energy outward through the objective lens toward the target. It was postulated that the sensitivity of the NTS"A" CCD's could possibly provide the capability to view the downrange location of the onboard laser designator spot--a tremendous asset to the operator for precision targeting and increasing laser guided weapon lethality. Tube launched, optically tracked, wire guided (TOW) missile tracking is accomplished by a near IR tracker integrated in both the NTS and NTS"A" NFOV optical trains. As such, the optics are filtered in the near IR wavelengths to improve TOW missile tracking performance at extended ranges.

\section{SCOPE AND METHOD OF TEST}

The performance of the NTS"A" TVC was measured via direct comparison to the DVO of the basic NTS during ground based and flight testing. The metric for evaluation in both phases of testing was contrast sensitivity. Until recently, most measurements of visual performance have involved either size or contrast, but not both. With a few exceptions, researchers measured either ability to resolve detail in small, high contrast targets, or to distinguish contrast in a very low-contrast, relatively large targets. The first of these abilities is usually referred to as visual acuity and the second as contrast threshold. Because the imagery display user must resolve edges that include a range of both sizes and contrasts, information on visual performance when both of these parameters are varied is much more useful in system performance [1]. The more detailed the target, the higher is its spatial frequency. Typical visual acuity targets such as Snellen letters or Landholt rings are high contrast, high spatial frequency stimuli. Measuring acuity with such targets reveals little about the operator's ability to see low contrast, low spatial frequency targets such as a car on a road at night or a person in a dark movie theater. In fact, contrast sensitivity has been found to be a better predictor than standard visual acuity of people's ability to discriminate highway signs and is a very good predictor of air-to-ground search performance [2].

During ground based testing, a 50 inch collimator was positioned directly in front of the sensor gimbal. The target utilized for ground based testing was the USAF 1951 Medium Power Resolution Target. Illumination levels were varied from below 10 lux (twilight) to over 18,000 lux (full daylight), while target contrast was varied in five increments. Several operators were sampled in the front seat of each aircraft to determine the contrast sensitivity of each sensor.

Quantitative inflight resolution testing was designed to validate static ground test results and ascertain the impact of airframe induced vibrations on the sensor operator's ability to resolve a target. Testing consisted of NTS"A" TVC flights versus a low contrast, tri-bar target (Fig. 7) with a frequency of 1.093 cycles/meter, and a luminous contrast of 0.33 , where luminous contrast is given by Eqn. (1):

$$
\text { Luminous contrast }=\frac{L_{\max }-L_{\min }}{L_{\min }}
$$

where $L_{\max }$ and $L_{m i n}$ are the maximum and minimum luminances in the pattern, 0.4 and 0.3 , respectively.

Flights were conducted in periods of high (direct sunlight) and low (overcast) illumination levels. The aircraft was maneuvered to a position whereby the target, but not the bars, was visible and normal to the sensor in narrow field of view (NFOV). Slant range was gradually decreased until three distinct bars were detected. Slant range to and luminance at the target were recorded while the sensor was toggled to the next higher field of view and the process repeated through the three optical fields of view. Helicopter indicated airspeed was incremented from 60 KIAS to 120 KIAS on subsequent data runs to vary the magnitude of airframe vibrations.

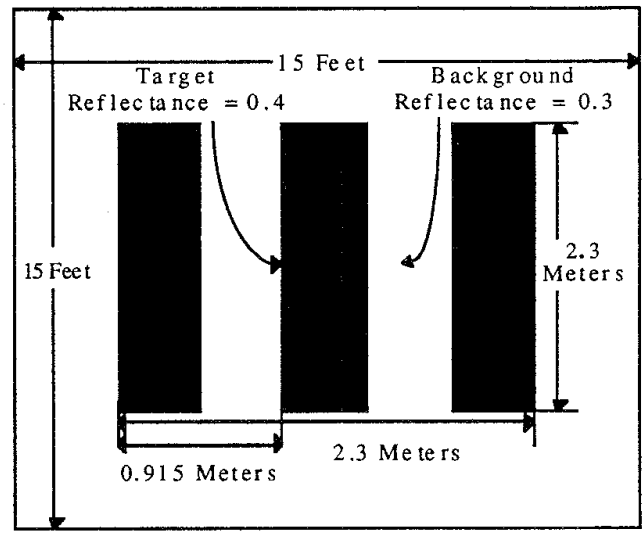

Figure 7. Low Contrast Resolution Target

Qualitative inflight testing consisted of direct side-by-side comparative testing between the NTS and the NTS"A" versus vehicular targets in a tactical background. Various tanks, armored personnel carriers, and military utility vehicles were positioned, in turn, in a known target location. The two aircraft were flown in formation a sufficient distance from the target area such that the presence or movement of a vehicle was undetectable in even the highest power magnification. A target vehicle unknown to the system operators was then placed in view in the center of the target area. Beginning in NFOV, the two aircraft were flown side by side in tight formation while steadily decreasing slant range toward the unknown target. Detection, recognition, and identification distances were recorded from both aircraft on each data run. Detection was defined as the presence of a potential target demanding attention. Recognition was defined as the point where the object was distinguished from similarly classed military objects, e.g., tank versus APC. Identification was defined as the point where the target's specific military designation was discernible, e.g., M-60 versus T-72 [3]. Data runs were 
conducted in conditions of bright sunlight, overcast skies, and at dusk.

\section{Data Analysis}

Data from quantitative ground and flight tests versus resolution targets were reduced and generalized into minimum resolvable contrast (MRC) curves. The data were unreferred to predict minimum detection ranges for various illumination conditions and spatial frequencies for a given prevailing visibility. Data from qualitative flight tests versus tactical targets was analyzed based on the experiments performed by Johnson in 1957. The results of this experiment, now known as the "Johnson Criteria" are shown in Table 2. In essence, Johnson placed a set of tribar patterns next to a military target as he varied conditions. He noted what tri-bar frequency could just be resolved when a given discrimination task (detection, recognition, and identification) could just be accomplished. Johnson related visual discrimination levels to the Air Force tri-bar target frequencies using the minimum dimension of the target as a reference. From Table 2 it can be seen that identifying a truck was found equivalent to resolving, under the same conditions, a bar chart whose bars were one sixteenth $(1 / 16)$ the minimum dimension of the truck. (Note that 8 line pairs are equal to 16 bars on a test target.) [3].

Table 2. The Johnson Criteria

\begin{tabular}{|c|c|c|c|}
\hline \multirow{2}{*}{$\begin{array}{c}\text { Target } \\
\text { Broadside) }\end{array}$} & \multicolumn{3}{|c|}{$\begin{array}{c}\text { Resolution per Minimum Dimension } \\
\text { (Line Pairs) }\end{array}$} \\
\cline { 2 - 4 } & Detection & Recognition & Identification \\
\hline \hline Truck & 0.9 & 4.5 & 8.0 \\
\hline M-48 Tank & 0.7 & 3.5 & 7.0 \\
\hline Stalin Tank & 0.75 & 3.3 & 6.0 \\
\hline Centurion Tank & 0.75 & 3.5 & 6.0 \\
\hline Half-Track & 1.0 & 4.0 & 5.0 \\
\hline Jeep & 1.2 & 4.5 & 5.5 \\
\hline Command Car & 1.2 & 4.3 & 5.5 \\
\hline $\begin{array}{c}\text { Soldier } \\
\text { (Standing) }\end{array}$ & 1.5 & 3.8 & 8.0 \\
\hline $105 m m$ Howitzer & 1.0 & 4.8 & 6.0 \\
\hline \hline Average $\left(\mathrm{N}_{\mathrm{J}}\right)$ & $1.0 \pm 0.25$ & $4.0 \pm 0.8$ & $6.4 \pm 1.5$ \\
\hline
\end{tabular}

After computing the observed area of the target $A_{T}$ in square meters given the vehicle dimensions, the spatial frequencies were calculated by determining the critical dimension $\left(D_{c}\right)$ in meters of the target to be resolved, where

$$
\mathrm{D}_{\mathrm{c}}=\sqrt{A T} \text { (meters) }
$$

By applying the Johnson criteria to the critical dimension of the test targets, $D_{c}$, the spatial frequency is given by

$$
S F=\frac{R}{2 X}(\text { cycles } / \mathrm{mrad})
$$

where $R=$ range to the target in kilometers and $X=$ the dimension to be resolved in meters given by

$$
X=\frac{D_{c}}{2 N J} \text { (meters) }
$$

where $N_{J}$ is the average number of line pairs required to perform each discrimination task (Table 2). The specific vehicles used in the evaluation are listed in Table 3 along with the associated spatial frequency per kilometer required to perform the various discrimination tasks.

Table 3. Target Spatial Frequencies

\begin{tabular}{|c|c|c|c|}
\hline \multirow{2}{*}{ Vehicle } & \multicolumn{3}{|c|}{$\begin{array}{c}\text { Spatial Frequency per km } \\
\text { (cycles/mrad) }\end{array}$} \\
\cline { 2 - 4 } & Detection & Recognition & Identification \\
\hline \hline $\begin{array}{c}\text { M-60 Rise Passive } \\
\text { Tank }\end{array}$ & 0.30 & 1.21 & 1.94 \\
\hline $\begin{array}{c}\text { M-2 Bradley } \\
\text { Armored Fighting } \\
\text { Vehicle }\end{array}$ & 0.34 & 1.37 & 2.19 \\
\hline $\begin{array}{c}\text { M-551 Sheridan } \\
\text { Tank }\end{array}$ & 0.38 & 1.50 & 2.40 \\
\hline $\begin{array}{c}\text { M-548 Tracked } \\
\text { Ammunition Carrier }\end{array}$ & 0.37 & 1.47 & 2.36 \\
\hline $\begin{array}{c}\text { M-813 5 Ton } \\
\text { Wrecker }\end{array}$ & 0.35 & 1.38 & 2.21 \\
\hline HMMWV & 0.46 & 1.83 & 2.93 \\
\hline $\begin{array}{c}\text { M-113 Armored } \\
\text { Personnel Carrier }\end{array}$ & 0.53 & 2.13 & 3.41 \\
\hline
\end{tabular}

Luminance at the target was measured during flight tests with a portable light meter calibrated in kilofootLamberts (kfl). To maintain consistency in the comparison of ground test data in units of illuminance (lux) to flight test data in units of luminance ( $\mathrm{kfL}$ ), a conversion was necessary to determine the approximate illuminance of the target. If the target area is assumed to be a Lambertian surface, then the luminous exitance $\boldsymbol{M}$, or luminous flux output per unit surface area, in lumens per square meter (equivalent to lux) is

$$
M=\pi L \quad\left(1 \mathrm{~m} / \mathrm{m}^{2}\right)
$$

where $\mathrm{L}$ is the luminance in lumens per square meter and steradian $\left(\mathrm{lm} / \mathrm{m}^{2} \mathrm{sr}\right)$ or nit $(\mathrm{nt})$. The Electro-Optics Handbook [4] gives a conversion factor of $3.426 \mathrm{ftL}$ per nit, which when entered into Equation (5) gives

$$
1 \mathrm{ftL} \cong 10.76 \operatorname{lux}
$$

Prior to generalizing the static ground test or "laboratory" MRC data, a correction for the atmospheric attenuation of observed contrast was applied. The transmittance of the atmosphere over a path length $R$ for very narrow wavelength bands may be expressed by

$$
T_{a}=e^{(-\sigma R)}
$$

where $\sigma$ is the spectral attenuation coefficient or "extinction coefficient." [4] 
Visibility refers to the horizontal distance $R_{V}$ for which the contrast transmission $\left(\mathrm{C}_{\mathrm{R}} / \mathrm{C}_{0}\right)$ of the atmosphere in daylight is two percent, where $C_{0}$ is the inherent contrast of a target and $C_{R}$ is the apparent contrast at range $R$. The effective value for $\sigma$ in Equation (7) for the visible spectrum of wavelengths, $\sigma_{\mathrm{V}}$, is dependent upon $\mathrm{R}_{\mathrm{V}}$

$$
\sigma_{\mathrm{V}}=3.912 / \mathrm{R}_{\mathrm{V}}
$$

where the units of $\sigma_{V}$ are determined by the units of $R_{V}$. [4] It has been shown that the apparent contrast $C_{R}$ reduces exponentially with range $\mathrm{R}$ in accordance with Equation (7), such that

$$
C_{R}=C_{0} e^{(-\sigma v R)}
$$

Equation (9) was used to account for the effects of the atmosphere on static MRC.

\section{RESUltS AND Evaluation}

\section{General}

The specific visual discrimination performance of the NTS"A" has been classified by the U. S. Government as CONFIDENTIAL. General, nondimensional comparisons between the two systems will be sufficient to describe the performance of the NTS"A".

\section{Minimum Resolvable Contrast}

Minimum resolvable contrast data gathered from ground testing versus the Air Force tri-bar target are presented in Figure 8.

The NTS"A" CCD camera significantly outperformed the DVO in low illumination conditions as evidenced by the large disparity in resolved spatial frequency in deep twilight conditions. The performance gap narrowed with increasing illumination to the point where DVO outperformed the CCD in illuminations commensurate with overcast skies. Data gathered at higher light levels than those depicted in Figure 8 indicated an additional increase in DVO performance, while CCD performance remained essentially constant beyond overcast conditions.

\section{Effect of Vibrations on MRC}

The effect of aircraft vibrations on system MRC was evaluated during flights versus the tri-bar target depicted in Figure 7. The effect of vibrations appeared to generalize well, inducing an approximately $37 \%$ reduction in resolved spatial frequency in both the narrow and medium fields of view. Varying airspeed did not result in a wide distribution of observed spatial frequency. These data provided the first indications of the unreliability of static, ground test data to accurately predict system performance in dynamic conditions. The observed degradation was most likely due to the cumulative effects of the atmosphere and operator proficiency, in addition to the kinesthetic effects of aircraft vibrations.

\section{Visual Discrimination Range Performance Prediction}

Data from static MRC tests were reduced and generalized into range prediction curves for the various visual discrimination tasks. Equations (3) and (4) show the relationship between resolved spatial frequency and range to the target. Therefore, the MRC curves of a sensor can be converted to the "range" domain by selecting a task to be performed, which determines $\mathrm{N}_{\mathrm{J}}$; selecting a target, which determines $\mathrm{D}_{c}$; and then solving Equation (3) for $\mathrm{R}$. The resulting curves retain the same shape, yet their abscissa is now in the range domain.

Since these data were gathered in a laboratory environment, a correction for the effect of atmospheric attenuation on observed contrast $\left(\mathrm{C}_{\mathrm{O}}\right)$ was applied. Given an observed target contrast, $C_{o}$, Equations (7) through (9) were used to plot the degradation of $\mathrm{C}_{\mathrm{O}}$ as a function of range along with MRC. A prevailing visibility of $30 \mathrm{~km}$, or very clear day conditions [4], was selected for $R_{V}$ in Equation (8). Curves detailing this process for identification are presented in Figure 9, where the target selected was a generic tank, viewed from a beam aspect or $90^{\circ}$ orientation, with a $C_{0}$ of 0.3. A small angle approximation was used for the observed target area, $\mathrm{A}_{\mathrm{T}}$, to discount the observed target area due to the observer's elevation above the target. The dimensions of this generic tank, $6.9 \mathrm{~m}$ width by $3.3 \mathrm{~m}$ height, provided a critical dimension, $\mathrm{D}_{\mathrm{c}}$ of $4.77 \mathrm{~m}$, per Equation (2). The value chosen for $C_{0}$ was determined to be representative for a military vehicle with a tactical paint scheme.

Range prediction as a function of ambient light level was accomplished by determining the intersection of each MRC curve (or its extrapolation) with the atmospheric attenuation curve. Generalized range prediction curves for detection, recognition, and identification of a tank, with a prevailing visibility of $30 \mathrm{~km}$, were generated for each system's various fields of view. Identification range prediction curves are presented for the NTS"A" TVC MFOV and NFOV and the NTS DVO NFOV in Figure 10. Predictions for detection forecasted a nearly constant superiority of the NTS"A" over the NTS across all light levels, with an average increase in detection distance of $62 \%$. For recognition, a steadily decreasing performance gap of the NTS"A" over the NTS was predicted with increasing light level, with the largest disparity, 67\%, observed in deep twilight conditions. Figure 10 shows a steadily decreasing identifiation performance gap, with the largest disparity, $72 \%$ in this case, also observed in deep twilight conditions.

\section{Target Detection, Recognition and Identification}

In general, the NTS"A" was able to detect a tank sized target in background clutter (low contrast) at $25 \%$ greater ranges than the DVO of the basic NTS in their respective narrow fields of view during high illumination conditions. Recognition and identification distances were improved by $8 \%$ and $16 \%$, respectively, versus the same targets. The marked improvement of the NTS" A" was more evident in low illumination conditions. The NTS"A" was able to detect a tank sized target in background clutter (low 

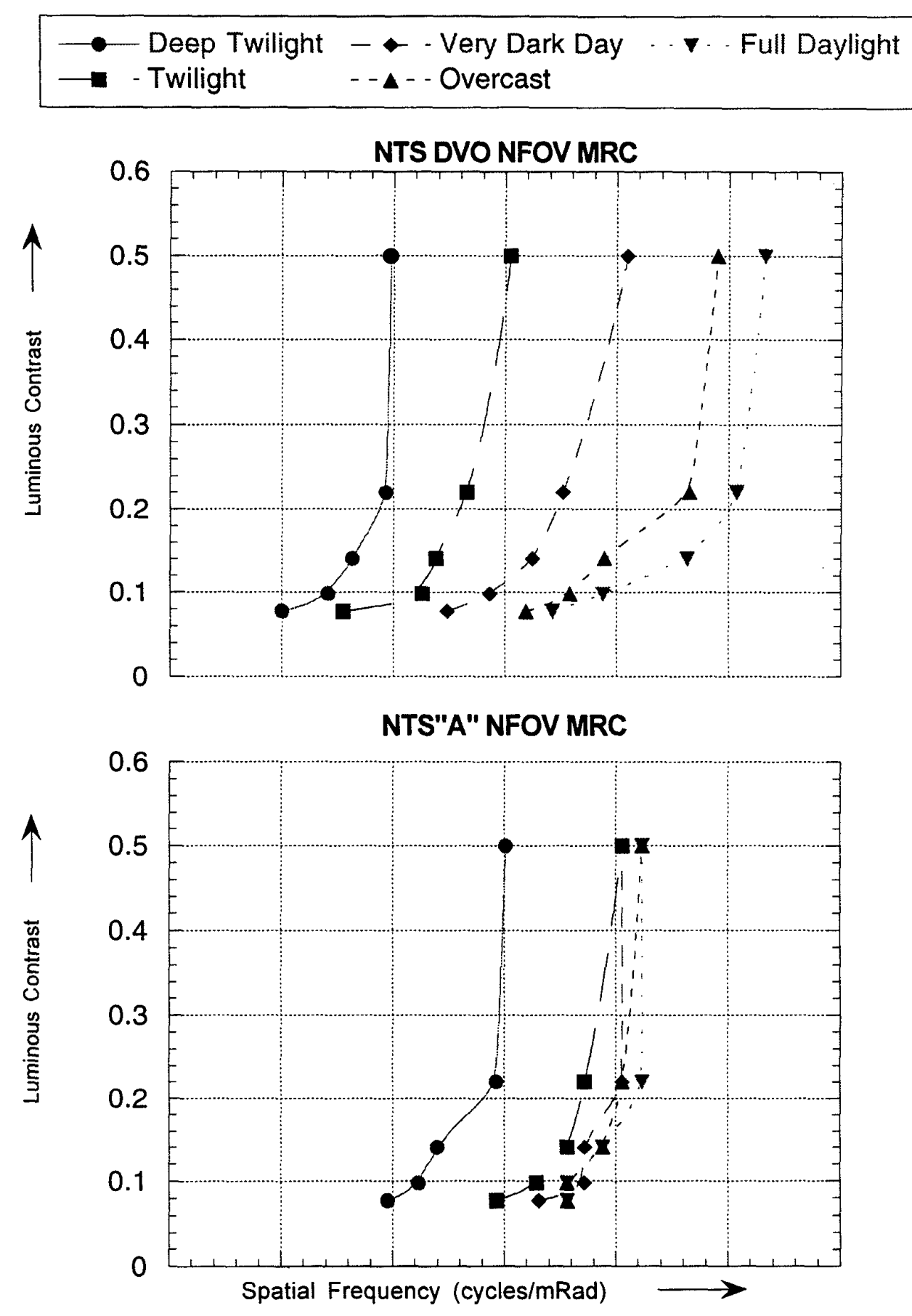

Figure 8. Narrow Field of View Minimum Resolvable Contrast (Static Conditions) ${ }^{1}$

Spatial frequency axis of both plots is same scale. Light levels defined as follows: Quarter Moon $=10^{-2}$ lux; Full Moon $=0.1$ lux; Deep Twilight $=1$ lux; Twilight $=10$ lux; Very Dark Day $=10^{2}$ lux; Overcast Day $=10^{3}$ lux; Full Daylight (Not Direct Sunlight) $=1-2 \times 10^{4}$ lux. [4] 


\section{NTS DVO NFOV Range Prediction Identification of a Tank \\ (30 km Visibility)}

(Basis: Static MRC Data)

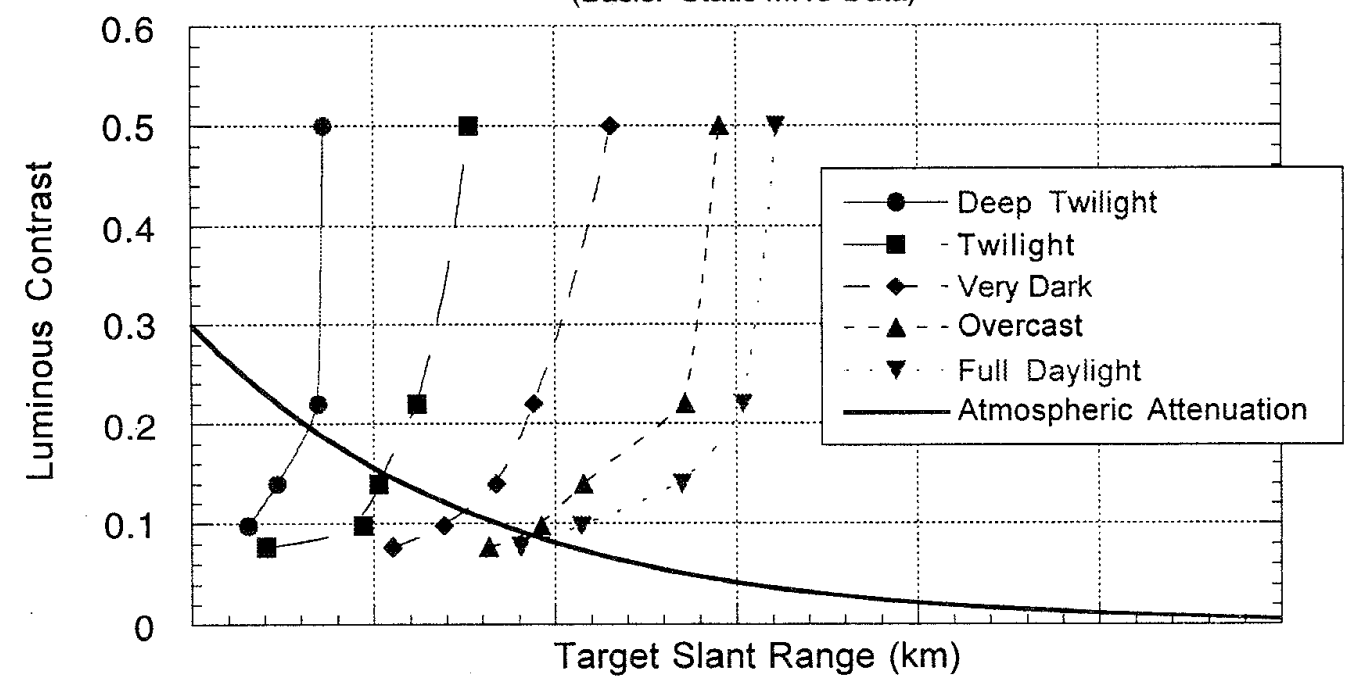

\section{NTS"A" TVC NFOV Range Prediction Identification of a Tank (30 km Visibility)}

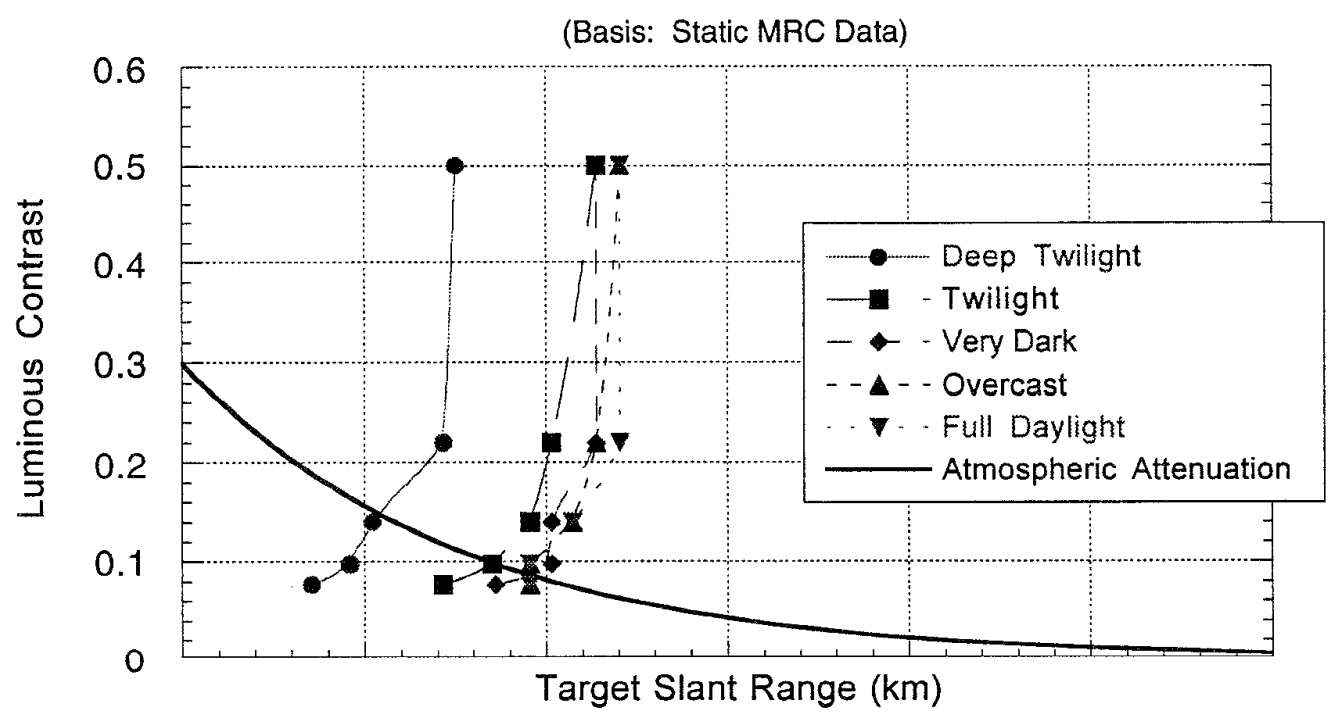

Figure 9. Effect of Atmosphere on NFOV MRC for Identification of a Tank (30 km Visibility) ${ }^{2}$

2 Slant range axis of both plots is same scale. Light levels as defined in Figure 8. 


\section{Range Prediction: NTS vs. NTS"A" \\ Identification of a Tank \\ (30 km Visibility)}

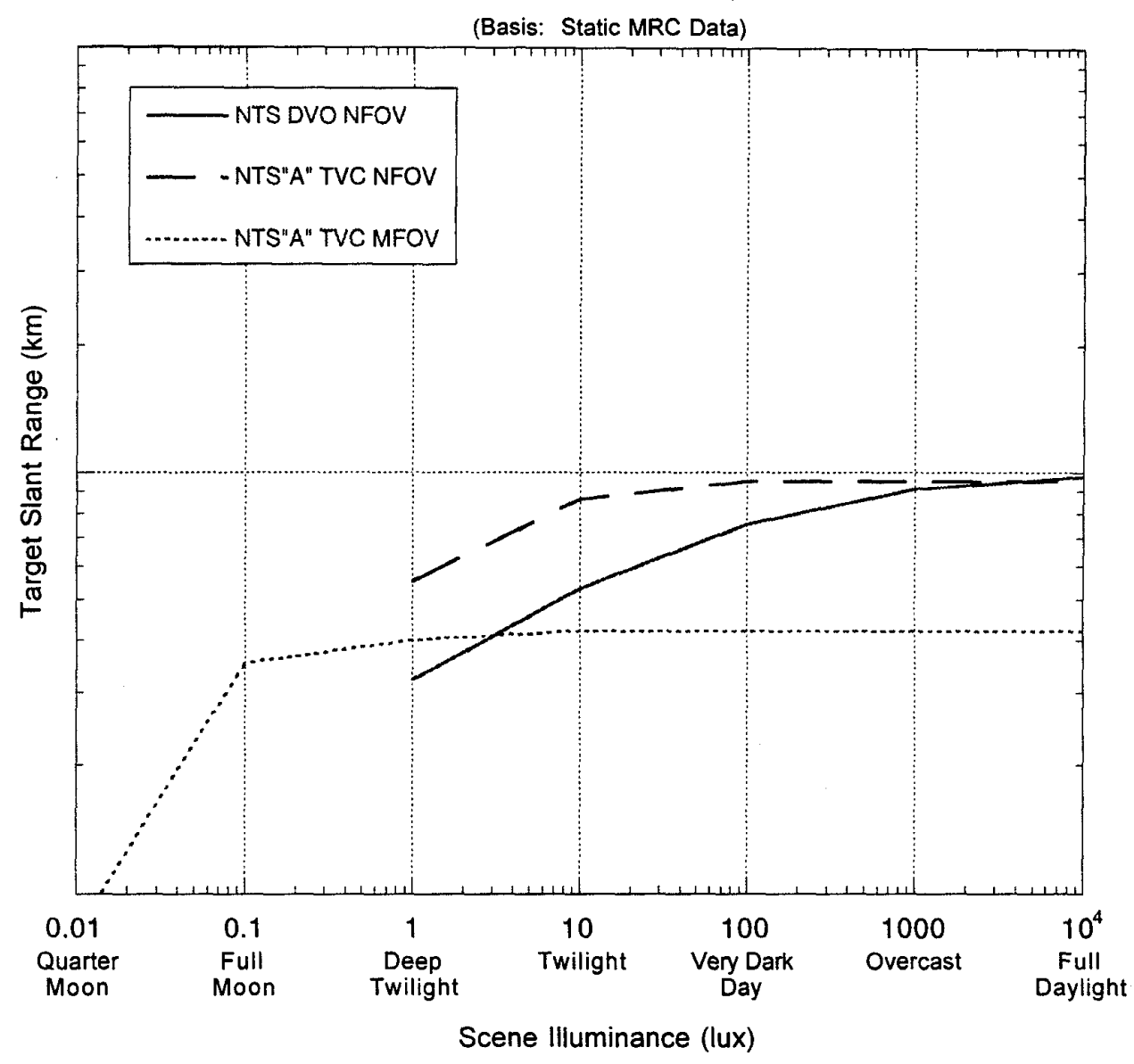

Figure 10: Range Prediction for Identification of a Tank (30 km Visibility)

contrast) at an average of over $200 \%$ greater ranges than the DVO of the basic NTS during low illumination conditions. Average recognition and identification distances were improved by $55 \%$ and $60 \%$, respectively, versus the same targets. The NTS"A" operator was forced to revert to the MFOV for low illumination DRI runs due to the degraded performance of the NFOV in civil twilight. The NTS"A" was capable of performing target detection, recognition and identification at tactically feasible distances versus vehicular targets with the sun up to $6^{\circ}$ below the horizon (civil twilight) with a measured luminance of $3 \mathrm{ftL}$. In the same conditions, the NTS DVO was totally unusable.

The filtering of near IR wavelengths in the NFOV optics due to the integration of the TOW missile near IR tracker did not affect the operator of the DVO because the eye is not sensitive to that region of the EM spectrum. Conversely, the NTS"A" NFOV was adversely affected as witnessed by the overall reduction in display brightness when compared to the MFOV, especially in low illumination conditions.
The MFOV optical path contains the fewest optics en route to a CCD and thus provided the sharpest display in all daytime light levels.

The general trends of the vibration corrected DRI predictions generated from static MRC data coincided well with the flight test data in illumination conditions down to overcast. The NTS"A" maintained superior DRI performance throughout all visual discrimination tasks, with an ever decreasing performance gap with increasing illumination. In low illumination conditions, the model accurately predicted the vast superiority of the NTS"A" TVC over the NTS DVO in very low ambient light levels. None of these results were unexpected, based on the system design and the data gathered from static testing. In comparing the actual DRI ranges with the predictions, the model tended to significantly overestimate the visual discrimination capabilities of both sensors, suggesting that additional factors beyond simply vibrations were contributing. 


\section{NTS"A" Human Factors}

In high light level conditions, the higher magnification of the NTS"A" improved target detection ranges, but reduced target area acquisition time dramatically. Since this research was more experimental than descriptive, more control was placed on the visual discrimination range variable. The NTS"A" operator, limited to a monochromatic, five squareinch display had a difficult time quickly acquiring the target area, even though it was clearly visible with the unaided eye. The NTS operator utilizing DVO had virtually no problem transitioning his DVO sensor to the target area once sighted with the naked eye. Postflight debriefs revealed that although detailed, target area colors were not discernible in the DVO, the large object color reference provided excellent cues to orient the sensor toward the known target area. The NTS"A" operator was forced to utilize large, high contrast objects (e.g., light brown fields next to evergreen trees) to orient the sensor. Furthermore, the two dimensional image presented on the display provided poor depth cues resulting in frequently misjudged ranges and subsequent false alarms. The $0.9^{\circ}$ field of view of the NTS"A" NFOV, as compared to the $4.6^{\circ}$ field of view of the NTS DVO NFOV (Table 1), was often disorienting to the operator when switching from the $3.5^{\circ}$ MFOV. Pilots claimed a low level of confidence during detection with the NTS"A" due to an increased level of uncertainty about the area being viewed. Often times, the NTS"A" operator delayed his "detection" call until more visual information was available at a decreased slant range to avoid tracking a false target and wasting a data run

The end result was a higher stress level on the NTS"A" operator to quickly train the sensor on an area clearly seen by the unaided eye, but difficult to acquire on a system that could not provide similar visual stimuli (poor visual compatibility). Once both sensors were sighted in on the target area, the high magnification (in high illumination conditions) and near IR responsivity (in low illumination conditions) of the NTS"A" contributed to consistent, superior performance over the DVO of the basic NTS.

\section{Laser Spot Tracking}

The ability of the NTS"A" to detect the laser energy reflected from a tactical target was tested while laser designating vehicular targets at various ranges in high (bright sunlight) and low (dusk) illumination conditions. In high illumination conditions, the downrange laser spot was not visible in any of the NTS"A" fields of view. In low illumination conditions, the laser spot was clearly visible only in MFOV, but at limited ranges tactically unrepresentative for target engagement. The inability to view the laser spot in high illumination conditions was probably due to the effect of saturation. In the bright sunlit conditions present in midday, the dominant wavelengths are in the visible spectrum. The energy reflected from the laser designator, operating at $1064 \mathrm{~nm}$, was probably of much lesser magnitude than the high energy, 380 to $780 \mathrm{~nm}$ visible light reflected from the target. In low illumination conditions, however, the energy differential was probably much less, thus enabling the CCD to sense and display the near IR energy reflecting off the target. The reduced laser detection range may be attributable to atmospheric attenuation due to haze or humidity as well as the collective optical and electronic losses (modulation transfer function) as those near IR wavelengths are sensed, processed, and displayed. Furthermore, the CCD responsivity in the design specification peaks at approximately $700 \mathrm{~nm}$ and steadily decreases to zero at approximately $1100 \mathrm{~nm}$.

\section{CONCLUSIONS}

The NTS"A" demonstrated limited potential for the air to ground targeting mission. A cursory look at the specification for the NTS"A" containing numbers such as those shown in Table 1 would lead one to believe that the NTS"A" is a quantum leap from the NTS during air to ground target DRI tasks. While CCD technology has obvious benefits in this application, there are major human factors issues to be addressed in order to optimize the performance of this system. Target acquisition time, although not specifically tested, is a significant parameter to a pilot intent on locating and destroying an enemy armed with even a rudimentary surface-to-air capability. Extended time spent unmasked by terrain searching for a target increases the very vulnerability that this technology upgrade intends to reduce. This is clearly where design tradeoffs must be considered. Each picture element (pixel) of a monochrome $\mathrm{CCD}$ carries a piece of light information reflected from the target. Color CCD's must spread the information carried by a single monochrome CCD pixel among several pixels (e.g., red, green, blue) to generate the display of color to the operator. The end result for a given $C C D$ size is an overall reduction in resolution with color CCD's, which may serve to reduce target area acquisition time albeit at the expense of reduced DRI distances. Clearly, the movement away from DVO is mandated by the laser threat and emergency egress considerations. Further research may lead to an optimum arrangement, such as a hybrid device consisting of a color CCD camera head for wide field of view search and target area acquisition, and a higher resolution, monochrome CCD camera head for target area DRI. It is also clear that an increase of information into the DRI process will increase the operator's efficiency in performing this task. Integration of the targeting sensor with on board navigational equipment which can drive the sensor in azimuth and elevation to a user selected ground coordinate is one example. Regardless of the arrangement, the design of an air to ground targeting system must be influenced by the human factors of vision.

\section{REFERENCES}

[1] Richard J. Farrell and John M. Booth, Design Handbook for Imagery Interpretation Equipment, Boeing Aerospace Company, 1984, p. 3.1-1.

[2] Mark S. Sanders and Ernest J. McCormick, Human Factors in Engineering and Design, New York: McGrawHill, Inc., 1993, pp. 97, 98.

[3] Shumaker, David L., Wood, James T., and Thacker, Clinton R., FLIR Performance Handbook, DCS Corporation, pp. 2-1 to 2-6.

[4] RCA Corporation, Electro-Optics Handbook, 1974. 
Major Paul Schreck, USMC is currently stationed as a test pilot with the AH-1W Cobra Flight Test Team at the Naval Air Warfare Center Aircraft Division, Patuxent River, MD. Major Schreck previously served in Marine Light/Attack Helicopter Squadron 169 at Camp Pendleton, CA, where he completed several overseas deployments, to include combat in the Persian Gulf War. He has a

BSEE from the University of Florida, is a graduate of Class 107 from the U.S. Naval Test Pilot School, and is currently working toward a Master of Science in Aviation Systems from the University of Tennessee Space Institute. 